\title{
Large-scale formation of shish-kebab in water-assisted injection-moulded high-density polyethylene with a high-molecular weight
}

\author{
H YUAN ${ }^{1}$, Y PAN $^{1}$ and X LIU ${ }^{1,2, *}$ (1) \\ ${ }^{1}$ National Engineering Research Center for Advanced Polymer Processing Technology, College of Materials Science and \\ Engineering, Zhengzhou University, Zhengzhou 450002, People's Republic of China \\ ${ }^{2}$ Jiangxi Province Key Laboratory of Polymer Micro/Nano Manufacturing and Devices, East China University of \\ Technology, Nanchang 330013, People's Republic of China \\ *Author for correspondence (Xianhu.liu@zzu.edu.cn)
}

MS received 10 March 2018; accepted 25 June 2018; published online 7 February 2019

\begin{abstract}
To promote flow-induced orientation and reserve the formation of the shish-kebab structure, a modified injectionmoulding technology named water-assisted injection moulding (WAIM), was applied to provide twice the shear flow and a rapid cooling rate on high-molecular weight high-density polyethylene (HDPE) melt in a mould cavity. Large-scale distribution of highly oriented shish-kebab was successfully achieved in a WAIM sample with a high-molecular weight. More importantly, the high-orientation degree of lamellae (larger than 0.4) in the thickness direction for a high-molecular weight WAIM sample is $>60 \%$, which is much more than a conventional injection-moulded sample $(15 \%)$. This work provides a new insight to achieve injection-moulded products with enhanced oriented structures.
\end{abstract}

Keywords. Orientation; high-density polyethylene; shish-kebab; water-assisted injection moulding.

\section{Introduction}

From the time, the shish-kebab was first reported, it has attracted immense research interest because of its remarkable improvement of some physical properties and significant theoretical values [1-10]. However, although conventional injection moulding (CIM) is the most common and convenient polymer processing method for the fabrication of polymer products with different geometric profiles, it is still a great challenge to form rich shish-kebabs in injection-moulded products due to the relaxation of oriented molecular chains and the 'weak' shear flow [1-4]. A relatively small number of shish-kebabs can be found in the thin shear (skin) region of the sample. In this respect, several novel injection moulding techniques, including oscillation shear injection moulding, loop oscillating push-pull moulding and multi-melt injection moulding have been developed to prepare injection-moulded samples with highly oriented or numerous shish-kebabs [4-11].

Recently, a novel and promising polymer processing technology, that is, water-assisted injection moulding (WAIM) has been the focus of extensive attention not only for academic reasons, but also for its industrial applications [12-16]. Generally, it can be divided into three stages. The cavity is first partially or completely filled with a polymer melt, and then high-pressure water is injected into the core of the polymer melt. Lastly, water continues to pack the melt till the melt is solidified. So far, much effort has been made towards the WAIM technology. In our group, WAIM has been successfully used to suppress the relaxation of molecular chains and fabricate injection-moulded parts with shishkebabs [12-16], but it is just the first step towards reinforcement of polymer products. Apart from the emergence of shish-kebabs, the relative proportion of shish-kebabs in the polymer part is another major factor responsible for the mechanical properties of industrial products. Cao et al [4] claimed that polymers with a higher molecular weight are more sensitive to flow field than the ones with a lower molecular weight, and a clearly pronounced orientation in the form of shish-kebab is found in polymers with a higher molecular weight. Previous reports have confirmed that WAIM samples exhibit hierarchical crystalline morphology through the thickness, including a shish-kebab-structured skin region, a typically spherulitic intermediate region and a shish-like structured water channel region [14]. Nevertheless, the two oriented regions i.e., the skin and water channel regions are very thin $(<0.3 \mathrm{~mm})$ and the orientation is also very low $(<0.49)$. However, such unique structure of the WAIM sample reminds of the bamboo-like bionic structures. From the point of view of engineering, it has excellent mechanical properties (combination of strength and toughness) if the orientation is high enough. To systematically understand the relationship between shear intensity, molecular weight and flow-induced microstructure in WAIM as a 
long-term project, research on high-density polyethylene (HDPE) with series of molecular weights is one of the necessary subjects.

Therefore, in this work, the orientation of high-molecular weight HDPE obtained via WAIM was preliminarily investigated based on the previous work. For comparison, the orientation of the same material obtained by CIM was also studied. Since the large-scale distributed shish-kebabs in high-molecular weight HDPE moulded by WAIM have never been reported until now, our work provides a new opportunity to understand fundamental issues about the molecular weights, processing parameters and conditions of WAIM in the formation of shish-kebab as well as a useful way for high validity of shish-kebab formation in the prepared injectionmoulded products.

\section{Experimental}

Two kinds of HDPE (marked F600 and 5000S) with different molecular weights were used. F600 has a high weight-average molecular weight of $5.0 \times 10^{5} \mathrm{~g} \mathrm{~mol}^{-1}$ and molecular-weight distribution (MWD) of 3.6, while 5000S possesses a lowmolecular weight of $3.17 \times 10^{5} \mathrm{~g} \mathrm{~mol}^{-1}$ and MWD of 5.4. The barrel temperature from the hopper to nozzle was 180 , 210,240 and $220^{\circ} \mathrm{C}$. The other detailed processing parameters and devices are described elsewhere [14].

2D-small angle X-ray scattering (SAXS) measurements were carried out using a NanoSTAR-U (Bruker AXS Inc.) with $\mathrm{CuK} \alpha$ radiation source $(\lambda=0.154 \mathrm{~nm})$ at room temperature. The sample preparation for 2D-SAXS measurements are illustrated in figure 1a. The cross-section photographs of the WAIM samples at the middle are shown in figure $1 \mathrm{~b}$. Clearly, the residual wall of 5000S is thinner than that of F600, indicating that the residual wall increases with the increased molecular weight. The lamellar orientation parameter was
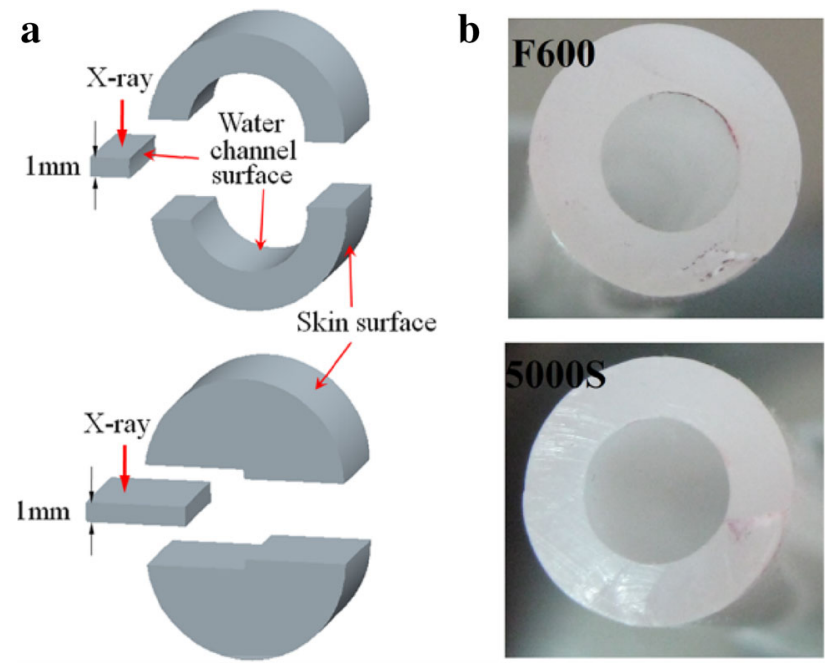

Figure 1. (a) Sample preparation for 2D-SAXS measurement and (b) cross-section of WAIM samples. calculated using the Herman's orientation parameter $f$ by the following equations [17]:

$$
\begin{aligned}
f & =\frac{3 \cos ^{2} \varphi-1}{2}, \\
\cos ^{2} \varphi & =\frac{\int_{0}^{\pi / 2} I(\varphi) \cos ^{2} \varphi \sin \varphi \mathrm{d} \varphi}{\int_{0}^{\pi / 2} I(\varphi) \cos ^{2} \varphi \sin \varphi \mathrm{d} \varphi},
\end{aligned}
$$

where $\varphi$ is the azimuthal angle between 0 and $360^{\circ}$ and $I$ the intensity. The orientation parameter $f$ has a value of unity when all the lamellae are oriented parallel to flow direction (FD), a value of -0.5 when all the lamellae are perpendicular to FD and a value of zero when there is a random orientation in the sample.

\section{Results}

Figure 2a shows the 2D-SAXS patterns of WAIM moulded F600 at an interval of $100 \mu \mathrm{m}$ from skin to water channel surface. It is observed that from 0 to $1300 \mu \mathrm{m}$ and 2500 to $2800 \mu \mathrm{m}$, two maxima along the equatorial direction are found. In other positions, the patterns show a very weak and broad equatorial maximum. Meanwhile, the meridional streak appears in the range from 0 to $400 \mu \mathrm{m}$ and from 2500 to $2800 \mu \mathrm{m}$. It is generally accepted that the simultaneous occurrence of meridional and equatorial maxima in the SAXS pattern can be attributed to the formation of the shish-kebab structure [18-20]. However, it is worth pointing out that the missing of meridional streak cannot be considered as the absence of shish [18]. In contrast, shish-kebabs are only observed from 0 to $700 \mu \mathrm{m}$ (denoted as the skin region) in the CIM sample (see figure $2 b$ ). Accordingly, figure 2 reveals a large-scale distribution of shish-kebabs along the thickness in the F600 WAIM sample.

Unexpectedly, for F600 WAIM sample, the equatorial maximum in the range from 900 to $1200 \mu \mathrm{m}$ becomes distinct again compared to those in the skin region. It is worth noting, however, that the equatorial maximum of the 2D-SAXS patterns for the WAIM samples only becomes distinct once along the thickness (except for the water channel region) as reported by us previously [14]. To our knowledge, this phenomenon has not been reported yet. Accordingly, figure 2 also indicates that the enhancement of shish-kebabs emerges (from the skin region to $1.4 \mathrm{~mm}$ ) in F600 WAIM sample.

To better understand the formation of shish-kebabs, the lamellar orientation parameters are estimated, and the results are shown in figure 3. Overall, the orientation degrees in WAIM samples are larger than those of CIM samples. It means that water penetration plays an important role in the enhancement of orientation, not only in the water channel region, but also in the skin region, which is in good agreement with our previously reported results [14-16]. Compared with 5000S, F600 has higher orientation degrees. This is consistent with the general consensus, i.e., it is much easier for a higher 


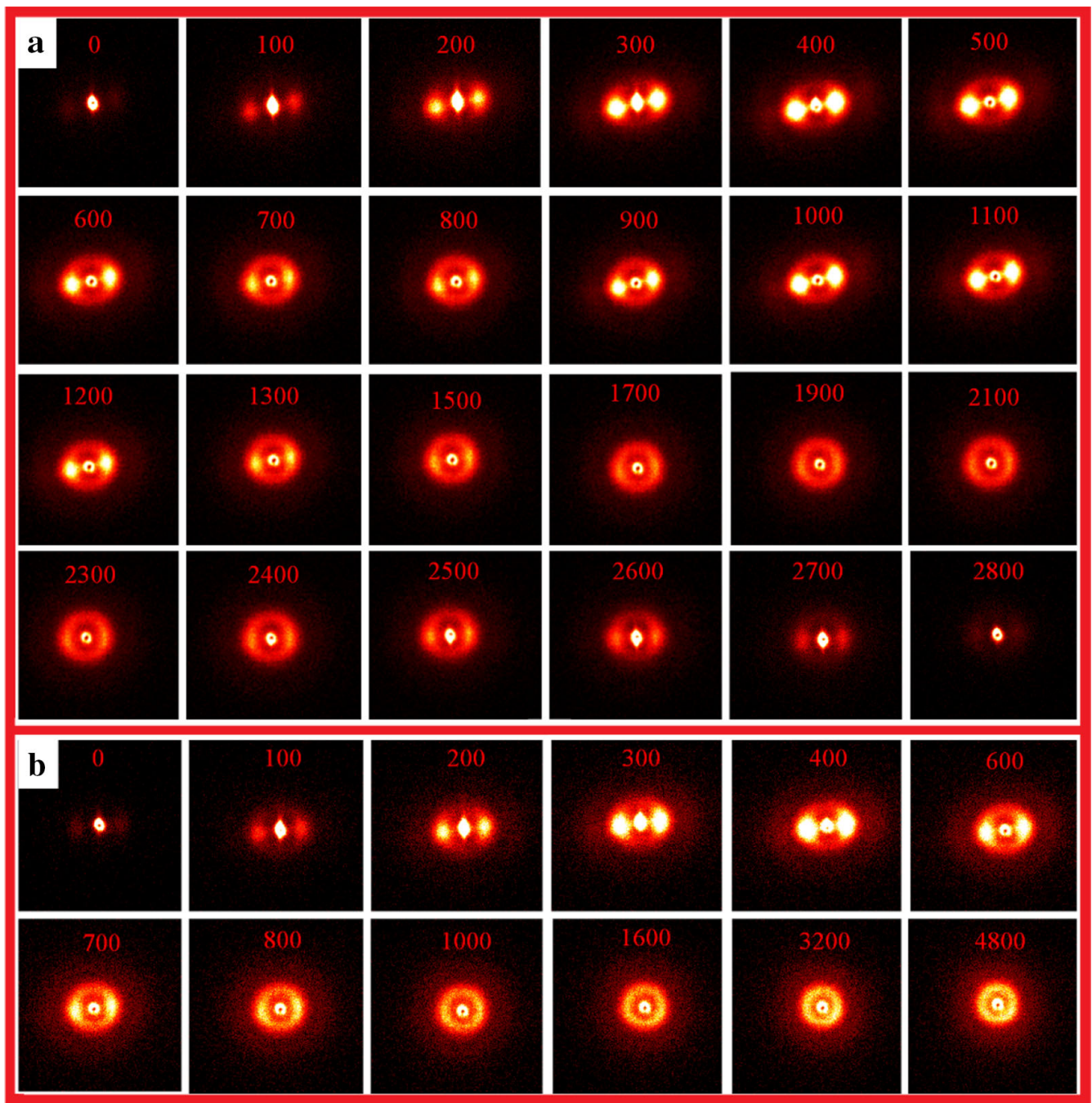

Figure 2. 2D-SAXS patterns of F600: (a) WAIM and (b) CIM samples. The flow direction is horizontal. The number (in $\mu \mathrm{m}$ ) on each image represents the distance away from the skin surface.

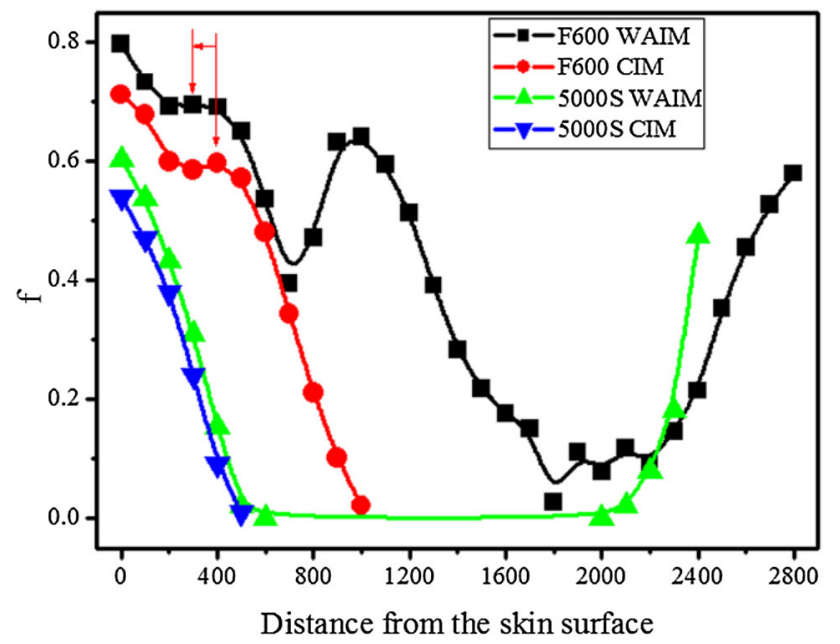

Figure 3. Lamellar orientation parameters of WAIM and CIM samples. molecular weight polymer to form preferential orientation in flow field than the lower molecular weight ones. Additionally, it is worth noting that the high-orientation degree of lamellae $(>0.4)$ in the thickness direction for F600 WAIM sample is $>60 \%$, which is much more than F600 CIM (15\%), 5000S WAIM (17\%) and 5000S CIM (6\%) samples.

On the other hand, from figure 3 , it was found that there is a shoulder peak (see the arrows) for F600 samples, which does not appear in 5000S indicating that the high-molecular weight is one of the important reasons for this phenomenon. F600 has a high viscosity, which is proportional to the molecular weight [18-21]. Thus, F600 is more viscous than 5000S. This is also documented in ref. [4,22], in which the HDPEs were the same as the ones in this study. Therefore, under the same cooling rate, the higher viscous melt is relatively difficult to flow, which is displayed in different viscosities, this can be confirmed by the residual wall of the WAIM sample shown in figure $1 \mathrm{~b}$. In addition, three kinds of HDPE with 
Table 1. Different water-injection pressures for different polymers (-: failure, + : success).

\begin{tabular}{lccc}
\hline & $5 \mathrm{MPa}$ & $10 \mathrm{MPa}$ & $15 \mathrm{MPa}$ \\
\hline HDPE F600 & - & - & + \\
HDPE 5000S & - & + & + \\
HDPE 2911 & + & + & + \\
\hline
\end{tabular}

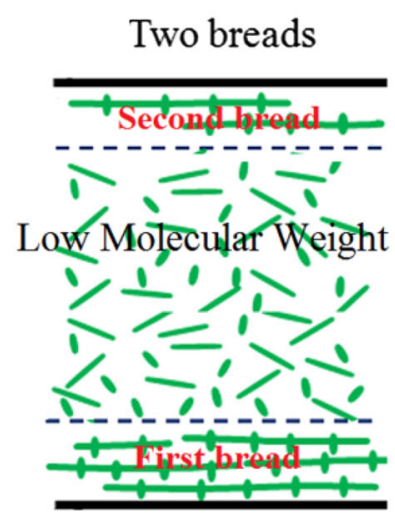

a Realized Part

\section{Two breads}

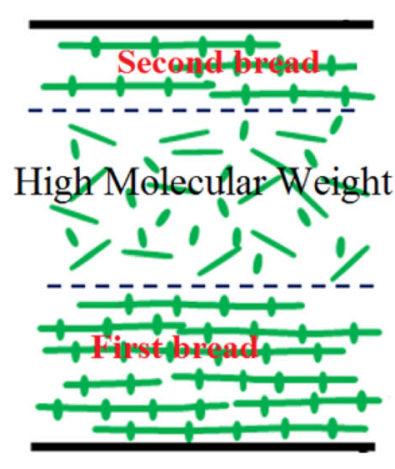

b Realized Part

\section{Three breads}

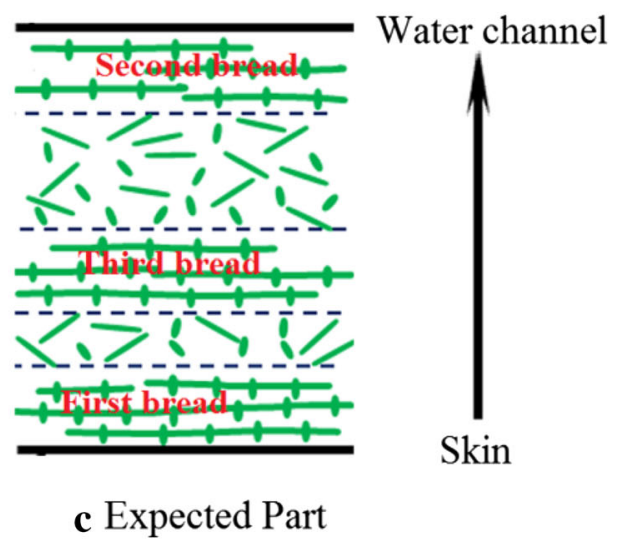

Figure 4. Schematic representing the sandwich-like structure in the different WAIM samples across the residual thickness. The flow direction is horizontal.

different molecular weights and different water injection pressures $(5,10$ and $15 \mathrm{MPa})$ were used. However, it was found that the required water-penetrating pressure in different polymer melts are different, and the results are shown in table 1 and then led to the stratified flow. This is the reason for the formation of multi-peaks for F600. Furthermore, one can note that the maximum shoulder peak value of CIM sample is around $500 \mu \mathrm{m}$ position, but it falls to $400 \mu \mathrm{m}$ for WAIM sample. This is mainly attributed to the fast cooling brought by the water penetration which suppressed the relaxation of shish-kebabs. Anyhow, the shoulder peak provides clear evidence for the formation of multi-peaks when another shear stress is involved. Therefore, this can be used for the analysis of the shish-kebabs around $1000 \mu \mathrm{m}$.

For the WAIM processes, the pressurized water is the only power to propel the melt forwards after the melt-filling process. In view of this, we conjecture that such obvious shish-kebabs around $1000 \mu \mathrm{m}$ should be attributed to the stratified flow caused by the water penetration. Additionally, the molecular chains for high-molecular weight species would be expected to possess much longer relaxation times than those of lower molecular weight species, and therefore can remain oriented after cessation of flow. It is thus expected that due to the higher heat capacity of water and aforementioned factors, large scale region of highly oriented shish-kebabs in highmolecular weight WAIM samples are preserved here.

The processing technique is an important factor that determines the macroscopic properties of prepared polymer materials in application [23-25]. However, the properties of a polymer product also depend on the polymer used and our capability to manipulate its internal morphology and structure [26-30]. Recently, a bamboo-like bionic structure has been reported [8,31] comprising a strengthened skin region with highly oriented shish-kebabs and a toughened core with an isotropic texture. This structure not only shows many desirable tensile properties, but also presents great impact property. In our case, a true meaning of a sandwich-bamboo-like bionic structure was obtained in the HMW WAIM sample. It shows a unique structure that contains a tough shell both outside and inside and soft material between the shells as a natural process of cross-disciplinary inspiration, most likely leading to some excellent mechanical properties in an energetically favourable manner. From this point of view, a larger number of bread slices of a sandwich-like bionic structure of WAIM sample has excellent mechanical properties than the two-bread slices sandwich structure. The possible formation of a sandwich-like bionic structure in the WAIM sample is schematically presented in figure 4. However, the major challenge in obtaining a multilayer structure is how precisely to control the processing parameters (such as the delay time of injected water) and to select the appropriate MW of polymer. Since the shishkebab formation can markedly improve many properties, naturally, it will be our future work to study the relationship between processing parameters and MW on the formation of shish-kebab. 


\section{Conclusion}

In this work, a large-scale distribution of highly oriented shish-kebabs was successfully achieved in an injectionmoulded high-molecular weight HDPE, for the first time, which was provided by a novel injection-moulding technology of WAIM. Our work could provide a promising doorway for this novel technology to achieve high performance products with some desirable physical properties in the light of manipulating the internal morphology and structure by selecting appropriate polymers and adjusting the processing parameters and conditions during the practical moulding process.

\section{Acknowledgements}

This work was supported by the Opening Project of Jiangxi Province Key Laboratory of Polymer Micro/Nano Manufacturing and Devices as well as by the HASTIT of Henan Province, China.

\section{References}

[1] Liu Z, Liu X, Zheng G, Dai K, Liu C, Shen C et al 2017 Polym. Test. 58227

[2] Liu X, Dai K, Hao X, Zheng G, Liu C, Schubert D et al 2013 Ind. Eng. Chem. Res. 5211996

[3] Pan Y, Guo X, Zheng G, Liu C, Chen Q, Shen C et al 2018 Macromol. Mater. Eng. 3031800083

[4] Cao W, Wang K, Zhang Q, Du R and Fu Q 2006 Polymer 47 6857

[5] Geng L, Li L, Mi H, Chen B, Sharma P, Ma H et al 2017 ACS Appl. Mater. Interfaces 921071

[6] Pan Y, Liu X, Shi S, Liu C, Dai K, Yin R et al 2016 Macromol. Mater. Eng. 3011468

[7] Li L, Li W, Geng L, Chen B, Mi H, Hoong K et al 2018 Chem. Eng. J. 348546

[8] Jiang J, Liu X, Lian M, Pan Y, Chen Q, Liu H et al 2018 Polym. Test. 67183
[9] Kuang T, Li K, Chen B and Peng X 2017 Composites, Part B 123112

[10] Liu X, Lian M, Pan Y, Wang X, Zheng G, Liu C et al 2018 Macromol. Mater. Eng. 3031700465

[11] Wang X, Pan Y, Qin Y, Voigt M, Liu X, Zheng G et al 2018 Polym. Test. 69478

[12] Liu X, Pan Y, Zheng G, Liu H, Chen Q, Dong M et al 2018 Macromol. Mater. Eng. 3031800035

[13] Liu X, Pan Y, Peng C, Hao X, Zheng G, Liu C et al 2016 Mater. Lett. 17219

[14] Liu X, Zheng G, Jia Z, Li S, Liu C, Zhang Y et al 2012 J. Appl. Polym. Sci. 1252297

[15] Zheng G, Jia Z, Liu X, Liu B, Zhang X, Dai Ket al 2012 Polym. Eng. Sci. $\mathbf{5 2} 725$

[16] Liu X, Zheng G, Dai K, Jia Z, Li S, Liu C et al 2011 J. Mater. Sci. 467830

[17] Picken S, Aerts J, Visser R and Northolt M 1990 Macromolecules 233849

[18] Zhu P and Edward G 2004 Macromolecules 372658

[19] Liu X, Pan Y, Zheng G and Liu C 2016 RSC Adv. 668969

[20] Liu Z, Liu X, Zheng G, Dai K, Liu C and Shen C 2015 J. Mater. Sci. 50599

[21] Lu Z, Pan Y, Liu X, Zheng G, Schubert D W and Liu C 2018 Mater. Lett. 22162

[22] Liang S, Yang H, Wang K, Zhang Q and Fu Q 2008 Polymer 56 50

[23] Shao Y, Yang Z, Deng B, Yin B and Yang H 2018 Polymer 140 188

[24] Luo F, Liu X, Shao C, Zhang J, Shen C and Guo Z 2018 Mater. Des. 14425

[25] Dou R, Shao Y, Li S, Yin B and Yang M 2016 Polymer 8334

[26] Liu X, Krückel J, Zheng G and Schubrt D 2014 Compos. Sci. Technol. 10099

[27] Qu M, Nilsson F, Qin Y, Yang G, Pan Y, Liu X et al 2017 Compos. Sci. Technol. 15024

[28] Liu X, Pan Y, Zheng G and Schubrt D 2016 Compos. Sci. Technol. 1281

[29] Liu X, Krückel J, Zheng G and Schubrt D 2013 ACS Appl. Mater. Interfaces 58857

[30] Pan Y, Liu X, Kaschta J, Hao X, Liu C and Schubert D 2017 Polymer 11334

[31] Xu H, Zhong G, Fu Q, Lei J, Jiang W, Hsiao B et al 2012 ACS Appl. Mater. Interfaces 46774 